\title{
3D cathodic protection design of ship hulls
}

\author{
L. Bortels ${ }^{1}$, B. Van den Bossche ${ }^{1}$, M. Purcar ${ }^{1}$, A. Dorochenko \\ \& J. Deconinck ${ }^{2}$ \\ ${ }^{1}$ Elsyca N.V., Zellik, Belgium \\ ${ }^{2}$ Vrije Universiteit Brussel, Department IR $\mid$ ETEC, Brussels, Belgium
}

\begin{abstract}
This paper presents a 3D software tool for the design and optimization of cathodic protection systems for submerged structures. It provides the corrosion engineer with a powerful tool for managing operational costs, significantly reducing expensive commissioning surveys and costly repairs, adding major value to the cathodic protection business.

The software is entirely CAD integrated such that it can deal with $3 \mathrm{D}$ CP-configurations of arbitrary complexity with parameterisation of all geometrical dimensions. The $\mathrm{CP}$ model is based on the potential model describing the ohmic drop in the electrolyte (soil, water) with non-linear boundary conditions that model the electrochemical reactions at anodes and cathodes.

In this paper, it is explained why the Finite Element Method is used to solve the problem. As an example the protection level of a hypothetical marine vessel using impressed current cathodic protection (ICCP) systems will be investigated. In addition, the underwater electric potential (UEP) of the vessel will be calculated.

Keywords: cathodic protection simulations, ICCP, advanced software package, marine vessel.
\end{abstract}

\section{Introduction}

Cathodic Protection (CP) systems are widely applied to buried and offshore structures. Most often, these CP systems contain a series of impressed current and/or sacrificial anodes, sometimes placed at a remote distance from the structure. The entire configuration of the $\mathrm{CP}$ system and the structures necessitate and justify the use of numerical simulations. 
First, the low accessibility of these structures makes installation, maintenance and repair very expensive. Also, the geometry of most steel structures that are subject to cathodic protection is too complex to allow analytical or even empirical estimations for the determination of the local protection level. Numerical modelling provides significant benefit by identifying insufficiently protected regions - possibly subject to corrosion, and overprotected regions subject to excess gas evolution and hence coating disbonding. As a consequence, numerical modelling allows simplification and optimization of installation, maintenance and repair. Moreover, models provide reference values for measurements on operational sites, enabling to trace and solve any possible anomaly.

Most of the publications dealing with the computation of the CP of both buried and submerged structures are based on the well known Boundary Element Method (BEM) [1].

Orazem et al. [2,3] use a 3D BEM approach to compute the protection level of large coating defects on pipelines. Results are presented for a pipe segment of limited length (10 feet), in presence of a parallel anode system. Riemer and Orazem [4] produced results for a larger pipeline $(>6 \mathrm{~km})$ with coating defects of varying size and investigated the ability of coupons in the vicinity of the defects to measure off-potentials. Adey [5] applied a full 3D approach to calculate the potential field in the neighbourhood of jacket joints under cathodic protection of sacrificial anodes. The present authors [6] used a 3D coupled multidomain BEM approach to simulate the protection level of a buried pipe segment surrounded by a concrete vault.

Aoki et al. [7] applied the BEM to detect a coating defect on a ship hull. DeGiorgi [8] made a significant contribution to the modelling work of the CP of ships. Diaz and Adey $[9,10]$ simulated the stray current corrosion of a vessel berthed to a steel dock protected by sacrificial anodes. The same authors presented a methodology based on boundary element techniques to determine the optimum anode configuration for shipboard ICCP systems.

The simulations presented in this paper are based on the Finite Element Method (FEM) [11], although it is well known that the Boundary Element Method (BEM) offers the advantage to reduce the dimensions of the problem and to obtain directly the current density distribution along electrodes.

\section{Mathematical model}

\subsection{The equations}

\subsubsection{The conducting medium}

CP protected structures (ships, tanks, pipes) are vast electrodes immersed in a semi-infinite electrolyte. Due to the nature of the conductive medium (soil, (sea)water) and its large scale, no concentration gradients occur in the electrochemical solution except for the well-known and very thin diffusion layer near the electrodes. Since the conducting medium is assumed to feature only charge transport with normal ohmic resistivity effects, the potential model holds, being described by the Laplace equation: 


$$
\bar{\nabla}(-\sigma \bar{\nabla} U)=0
$$

in which $\sigma$ is the conductivity and $U$ the potential field in the conducting medium. On insulating boundaries, the current density perpendicular to the surface is zero. The phenomena occurring in the diffusion layer and at the electrode interface are encompassed in the (non-linear) boundary conditions.

\subsubsection{Coated metal surfaces}

Protected structures are in general covered with a good quality coating. It is assumed that the latter can be described by a resistance that is placed in series with the pipe / soil polarisation resistance:

$$
j_{n}=f\left(V-U-E_{\text {corr }}+\rho_{\text {coat }} L_{\text {coat }} * j_{n}\right) .
$$

where $j_{n}$ is the local current density, $\rho_{\text {coat }}$ and $L_{\text {coat }}$ are the resistivity and the thickness of the coating respectively. $E_{\text {corr }}$ is the corrosion potential, $V$ and $U$ hold for the electrode and electrolyte potential respectively. Due to the high resistivity of the coating, curve (2) manifests a nearly linear behaviour.

Another approach to deal with coated surfaces is presented in equation (3). Here it is assumed that the polarisation of the surface is that of the metal. But a parameter $\theta$ is introduced that accounts for the fact that only a fraction of the total surface is active.

$$
j_{n}=\theta f\left(V-U-E_{c o r r}\right) .
$$

\subsubsection{Anodes}

The anodes are either sacrificial anodes or impressed current/potential electrodes. The electrode polarisation is often represented by an interpolation function of a measured electrode polarisation curve, relative to a mixed corrosion potential $E_{\text {corr }}$ :

$$
j_{n}=f\left(V-U-E_{\text {corr }}\right)=f(\eta) .
$$

\subsection{Numerical solution method}

As the conductivity of the computational domain is constant, in principle the BEM is to be preferred over other discretisation methods as Finite Element (FEM), Finite Volume (FVM) and Finite Difference Methods (FDM), to solve the simplified charge conservation equation (1).

The initial version of the software was based on the BEM which was for performance reasons soon replaced with the more advanced Fast Multipole Method [12]. Both methods have the advantage that only the boundaries of the 
3D model need to be meshed. The disadvantage however is that due to the intrinsic properties of both the BEM and FMM, the resulting system matrices remain quite populated, which severely limits the number of surface elements that can be used (about 40000 on a standard PC). Therefore, a complete new software code has been developed which is based on the well-known Finite Element Method (FEM) [13]. Using the FEM, the complexity of the problems that can be solved (i.e. the total number of surface elements) has been increased with at least an order of magnitude (when compared to FMM). The non-linear system equations are solved using a Newton-Raphson iterative method [14], combined with an advanced iterative linear solver to solve the resulting system of equations at each iteration. It is important to mention that when the Potential Model is solved using the FEM, the technical problem is rather a volume mesh generation problem, while a solver based on the BEM struggles more with matrix assembly and system inversion.

\subsection{Mesh generation}

In order to make the use the FEM possible, a powerful and automated 3D volume mesh generator is required that produces the meshes needed for the computations [15]. The developed mesh generator is fully CAD integrated, generates an unstructured grid and works in a hierarchical way. First the meshes (line segments) on all edges are generated, based on the obtained edge grid, all surfaces are triangulated and finally a volume grid of tetrahedral is generated based on the generated surface grid. All operations are controlled using a set of general parameters such as the targeted size of an edge, surface or volume element and the preservation of curvature. The size of an element can be locally different (see Figure 3) e.g. on electrodes more elements are generated than on insulating boundaries.

The unstructured surface and volume grid generators control the transition from the local mesh size to the targeted one, based on an interpolation/extrapolation functions and a user defined growing factor (see Figure 3).

\section{Description of the problem}

As an example, the cathodic protection level of a hypothetical marine vessel (catamaran type) using 4 separate impressed current cathodic protection (ICCP) systems, each delivering $3 \mathrm{~A}$, will be presented and investigated (see Figure 1).

The total length of the vessel is $120 \mathrm{~m}$, with a width of $40 \mathrm{~m}$ (waterline). Two ICCP systems are located in the middle (inside) of the catamaran, one at the port side (MP), the other one at the starboard side (MS). The other two ICCP systems are located at both sterns (outside) and are denoted as anodes SP and SS.

The resistivity of the seawater is assumed to be $0.25 \Omega \mathrm{m}$. The catamaran material used in the simulations is marine aluminium with polarization behaviour as measured by $\mathrm{Kim}$ et al [16]. The corresponding corrosion potential is -880 $\mathrm{mV}$ versus the $\mathrm{Ag} / \mathrm{AgCl}$ reference electrode. The coating of the catamaran is 
considered to be non-ideal. The coating defects are assumed to account for 1.0 percent of the total surface area. To that purpose, the polarization curve for the bare aluminium, as obtained in [16], has been scaled with a factor $\theta=0.01$ for the current density. The propellers are made from nickel, aluminium and bronze (NAB) and have a corrosion potential of $-200 \mathrm{mV}$ (versus $\mathrm{Ag} / \mathrm{AgCl}$ ). The polarization data are taken from the work by Hack [17]. It is assumed that the propeller and support are electrically continuous. The anodes are $7.5 \mathrm{~cm}$ in diameter and have a length of $1.5 \mathrm{~m}$. The anode material is a typical metal oxide for which the data have been taken from a proprietary database. A detailed view of the propeller and support with nearby anode is presented in Figure 2.
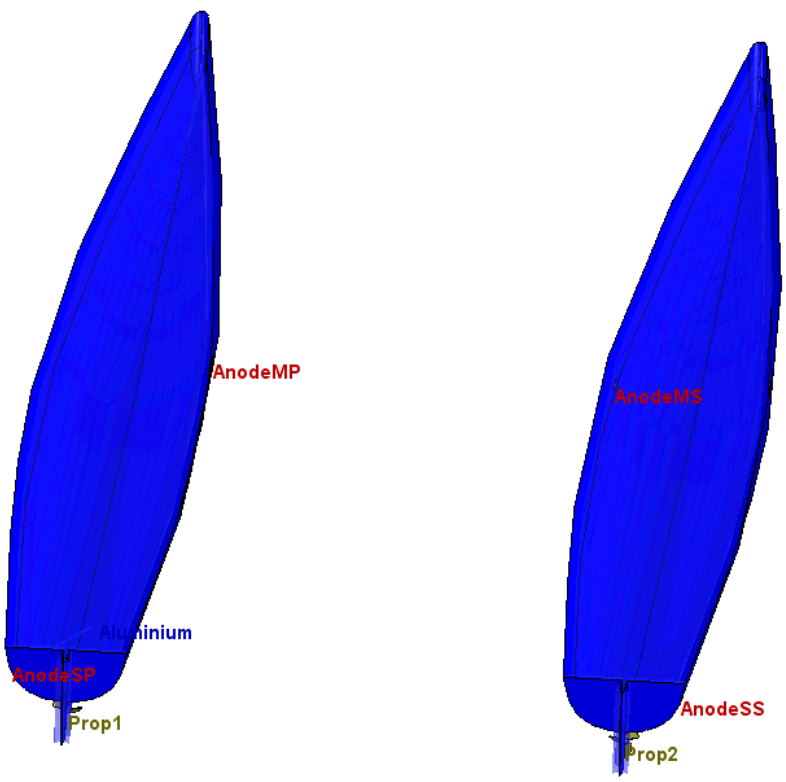

Figure 1: Layout of the catamaran with 4 ICCP systems: Anode MP, MS, SP, $\mathrm{SS} ;(\mathrm{M}=$ middle, $\mathrm{S}=$ stern; $\mathrm{P}=$ port, $\mathrm{S}=$ starboard $)$.

\section{Computational specifications}

The total number of triangular surface elements used to solve the problem is about 200000 . The corresponding tetrahedral volume mesh consists out of nearly 2500000 elements. There are in total about 500000 mesh points of which About 200000 points are located on surfaces. Details of the triangular surface mesh near one of the propellers and at waterline level is presented in Figure 3. The total calculation time on a $2.2 \mathrm{GHz}$ laptop is less than 6 minutes for 9 iterations in the Newton-Rapson procedure, after which a sufficient convergence level was reached. 
108 Simulation of Electrochemical Processes II

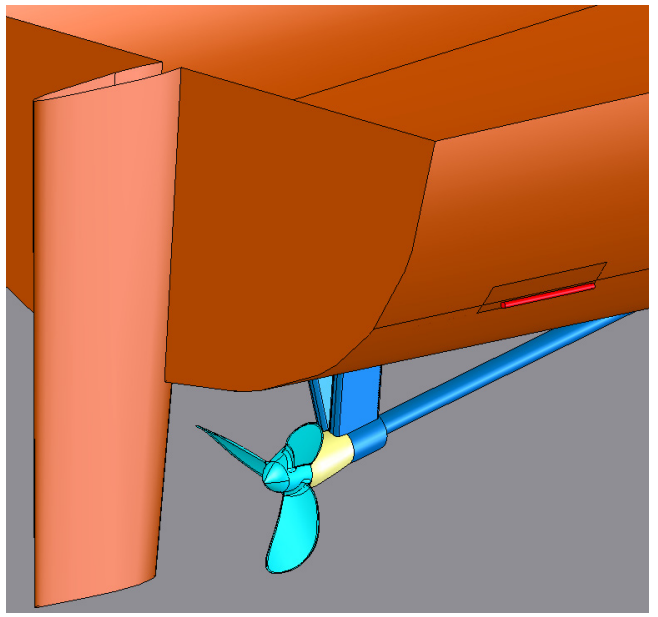

Figure 2: Detailed view of propeller and support with nearby anode (starboard site).
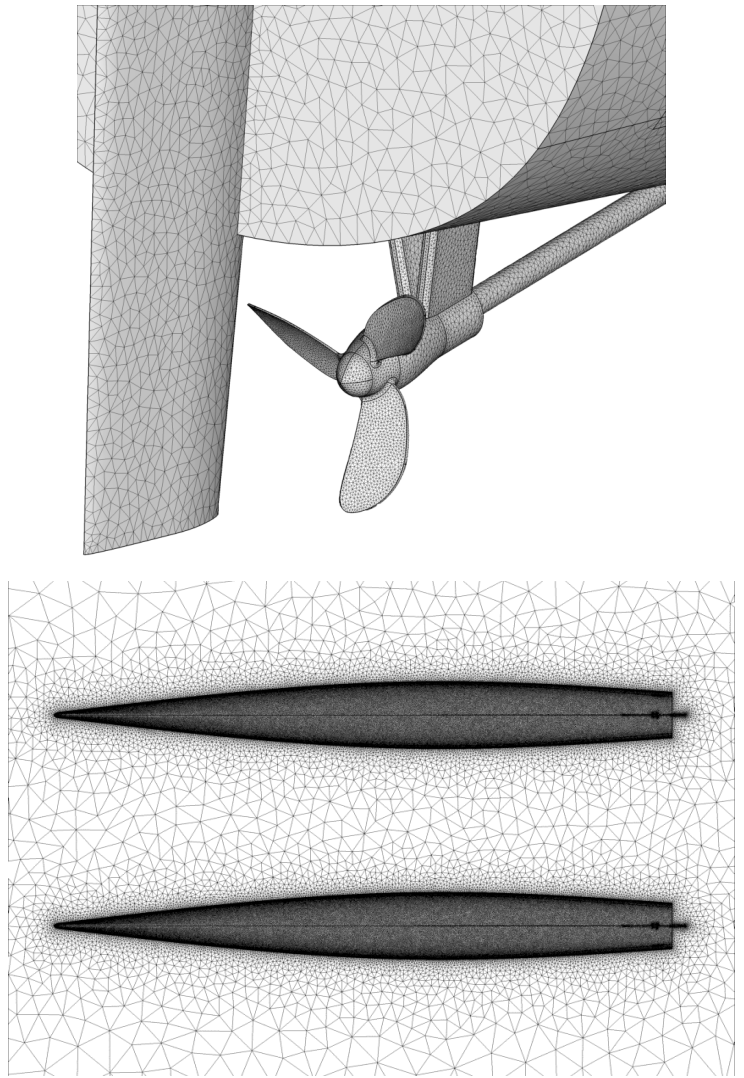

Figure 3: Zoom of surface mesh near one of the propellers (top) and at waterline level (bottom). 

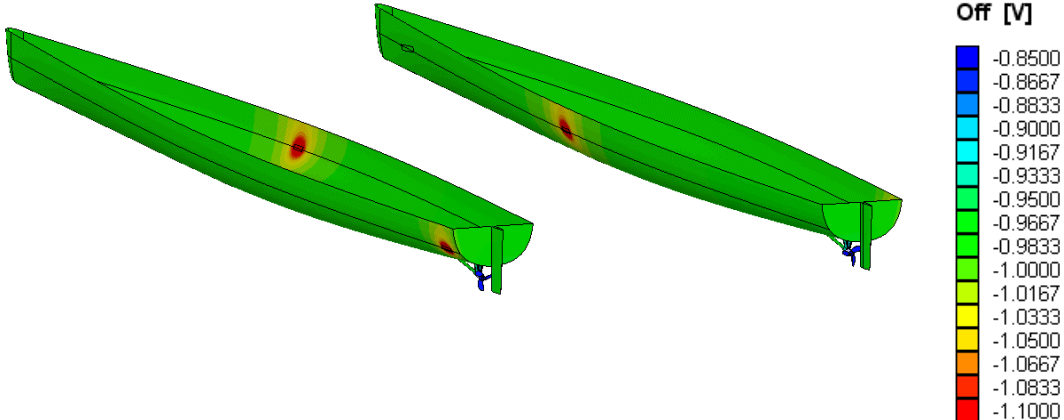

Figure 4: “Off” potential distribution along hull - normal operation.

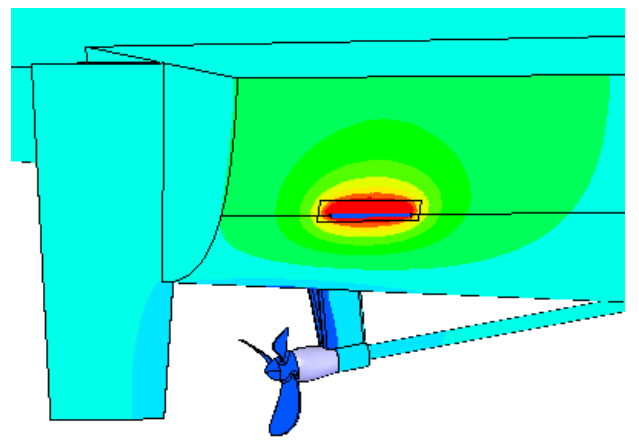

Off [V]
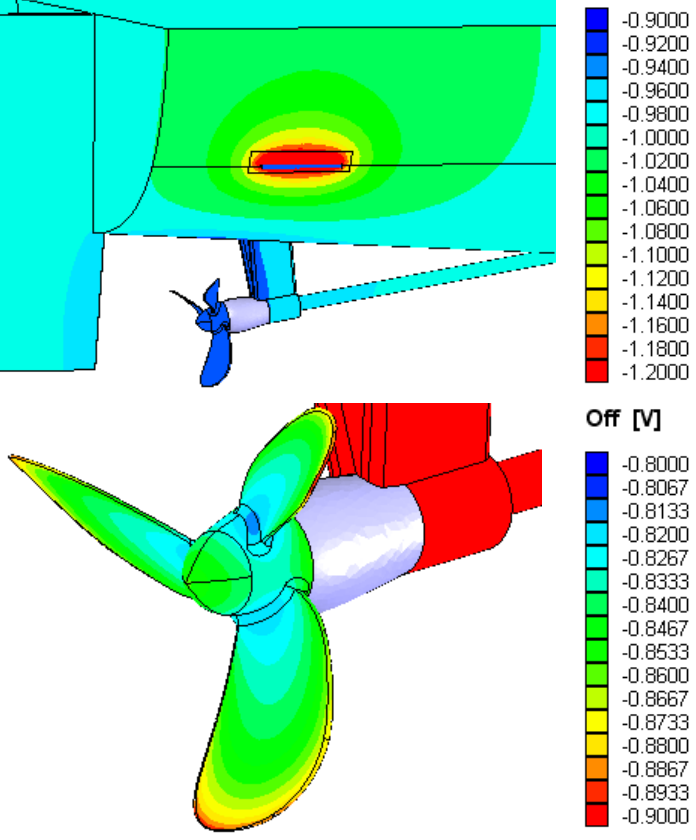

Off [V]

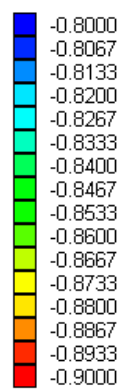

Figure 5: “Off” potential distribution near anode (top) and propeller (bottom) - normal operation.

\section{Results and discussion}

\subsection{Simulation of cathodic protection levels}

In this simulation the normal situation is investigated. All four ICCP systems are active delivering a total protection current of $12 \mathrm{~A}$. The calculated "off" potential 
distribution along the hull is presented in Figure 4. The average "off" potential is $-977 \mathrm{mV}$ which means that, taking into account the corrosion potential of $-880 \mathrm{mV}$, an average polarization of the hull of $-97 \mathrm{mV}$ is obtained.

Figure 5 (top) shows the "off" potential along the hull near the stern anode at starboard side. From this picture it can be observed that there is a slight overprotection of the hull directly near the anodes with "off" potentials more negative than $-1200 \mathrm{mV}$. Similar conclusions can be drawn from Figure 5 (bottom) presenting the calculated protection levels at the bare propeller. The obtained "off" potential at the hull near the propeller drops to $-930 \mathrm{mV}$ which means a polarization of only $-50 \mathrm{mV}$ which is below the minimum required limit of $-100 \mathrm{mV}$.

\subsection{Simulation of underwater electrical potential (UEP)}

Besides for optimisation purposes, the software can also be used to calculate the underwater electrical potential (UEP) of the catamaran. The calculation of the electrical field in the seawater around the vessel is straightforward as it can be obtained directly from the gradient of the potential obtained in the nodal points of the FEM volume mesh. Figure 6 shows the calculated x-component of the electrical field on a cutting plane $2 \mathrm{~m}$ below the keel.

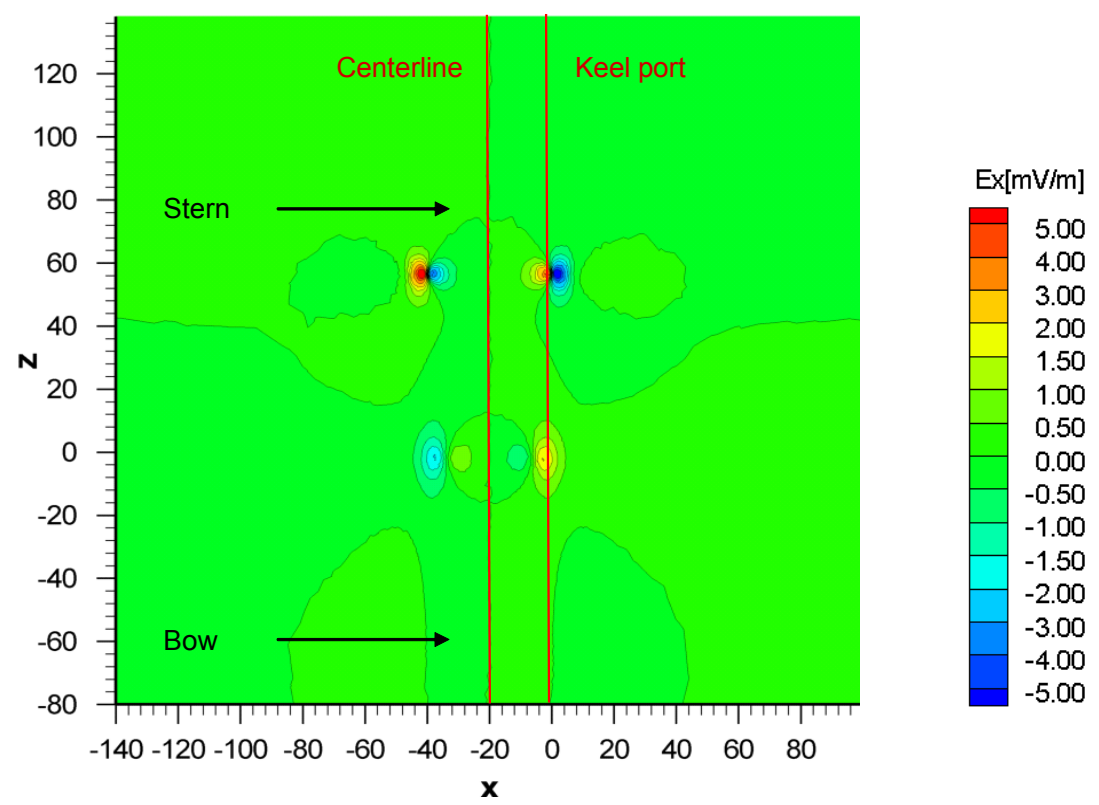

Figure 6: Underwater Electrical Potential UEP (Ex in $\mathrm{mV} / \mathrm{m}$ ) $2 \mathrm{~m}$ below keel. 


\section{Conclusions}

In this paper a 3D software tool for the design and optimization of cathodic protection systems for submerged and buried structures has been presented. The software is entirely CAD integrated, and is based on the combination of a powerful volume mesh generator and the Finite Element Method.

Using this software, the protection level of a hypothetical marine vessel using impressed current cathodic protection (ICCP) systems has been investigated in normal operation. Optimisation is a logical step.

The software has also been used to calculate the underwater electric potential (UEP) of the catamaran.

\section{References}

[1] C.A. Brebbia, Boundary Element Techniques - Theory and Applications in Engineering, Springer-Verlag Berlin, Heidelberg, 1983.

[2] M.E. Orazem, J.M. Esteban, K.J. Kennelley, R.M. Degerstedt, Corrosion 53 (1997) 427.

[3] M.E. Orazem, J.M. Esteban, K.J. Kennelley, R.M. Degerstedt, Corrosion 53 (1997) 264.

[4] D.P. Riemer, and M.E. Orazem, Corrosion 56 (2000) 794.

[5] R. Adey, Topics in Boundary Element Research, vol. 7, Electrical Engineering Applications, chapter 3, Springer-Verlag Berlin, Heidelberg 1990.

[6] M. Purcar, B. Van den Bossche, L. Bortels, J. Deconinck, P. Wesselius, Corrosion 59 (2003) 1019.

[7] S. Aoki, K. Amaya, K. Gouka, Optimal cathodic protection of ship, Boundary Element Technology XI, pp. 345-356, 1996.

[8] V.G. DeGiorgi, Boundary Elements XIX, pp. 829-838, 1997.

[9] S. Diaz and R. Adey. A Computational Environment for the Optimisation of CP system Performance and Signatures, Warship CP2001, Shrivingham, UK.

[10] S. Diaz, R. Adey, Boundary Elements XIX, pp. 475-485, 2002.

[11] Elsyca CPMasterV2.0, User Manual, www.elsyca.com.

[12] F. Korsmeyer, D. Yue, K. Nabors, J. White, Multipole-Accelerated Preconditioned Iterative Methods for Three-Dimensional Potential Problems, Proceedings of BEM 15, pp. 517-527, 1993.

[13] O. Zienkiewicz, The finite element method in engineering science, McGraw-Hill, London, 1971.

[14] J. Deconinck, Current distributions and Electrode Shape Changes in Electrochemical Systems, Lecture Notes in Engineering 75, Springer Verlag Berlin, ISBN 3-540-55104-2, 1992.

[15] Athanasiadis A.N., Deconinck H., Int. J. Numer. Meth. Eng. 58 (2003) 301 . 
112 Simulation of Electrochemical Processes II

[16] Y.-G. Kim, Y.-C. Kim, Y.-T. Kho, BEM application for thin electrolyte corrosion problem.

[17] H. P. Hack, Atlas of Polarization Diagrams for Naval Materials in Seawater, 1995. 